\title{
Tools and Techniques in Risk Identification: A Research within SMEs in the UK Construction Industry
}

\author{
Ali Rostami \\ Faculty of Science and Engineering, University of Wolverhampton, United Kingdom
}

Copyright $\subset 2016$ by authors, all rights reserved. Authors agree that this article remains permanently open access under the terms of the Creative Commons Attribution License 4.0 International License

\begin{abstract}
Risk identification plays a key role in the success of managing risk. Failure in the identification of risks can cause inadequacy in the whole process of risk management which led to non-achievement of organisational objectives. Tools and techniques facilitate the process of identification, and need to be adopted on the basis of firms' characteristics. The difficulty to recognise applicable tools and techniques within organisations is investigated as one of the key barriers that obstruct practise of risk management. This study discusses the efficacy of different tools and techniques of risk identification within Small and Medium Enterprises (SMEs) in the UK construction industry. Results from a questionnaire survey shows the challenges faced by SMEs in undertaking risk identification and highlights the most common techniques adopted among 453 organisations. Documentation review, expert judgment, checklist analysis and information gathering are seen as the most important techniques within risk identification; which are practised for their valuable results, uncomplicated processes and easy to understand structure. Conversely, the group-based techniques like brainstorming and Delphi techniques because of SMEs' inadequate level of knowledge and resources are less practised.
\end{abstract}

Keywords Risk Management, Risk Identification, Tools and Techniques, Construction Industry, Small and Medium Enterprises

\section{Introduction}

The Global Construction Perspectives and Oxford Economics [1] forecasted that the construction sector will grow by over $70 \%$ by 2025 . This significant growth in the global construction industry will create considerable opportunities for the UK construction organisations, but will equally generate demand for adequate reforms in project performance [2]. In order to improve the performance of the construction industry and take advantage of the new business environment, a number of studies have specified the key role of the risk management practice [3]. Chapman and Ward [4] explained that implementation and subsequent practise of risk management contributes to enhanced project performance. This view is also confirmed by the UK Government through the British Standard [5]. It states that the practise of risk management in organisational management resulted in the control of delays and budget overruns, which ultimately promotes the competitive advantage of organisations.

Studies in the UK construction industry presented the weak reputation of risk management within organisations [6] and [7]. Kim and Bajaj [8] and Frimpong et al. [9] investigated that the construction professionals' low level of familiarity with techniques and inability to elicit results of the processes are the most influential factors which impact the adoption of risk management in organisations. Chapman [10] and Couillard [11] added that, even professionals with frequent use of risk management have difficulty to understand the rational of its techniques' outputs in new projects. This difficulty is also compounded by SMEs' restricted resources which make both implementation and practice of risk management more complicated [12]. The take up and practise of risk management techniques involves a considerable level of investment which in some cases is unaffordable for SMEs. This investment signifies the high degree of effort required to understand and to learn how to use the techniques at both organisational and personal levels.

Tools and techniques are designed to facilitate processes and allow resources within an organisation to be applied efficiently to the activities which they are most suited. The adopted tools and techniques in both risk identification and risk analysis determine the accuracy of the results of the risk management process [13]. Failure in correct adoption of tools and techniques either in identification or analysis stages causes miscalculations in risk assessment, and critically impacts the organisational resources [14]. Adoption of tools and techniques within an organisation is significantly influenced by the internal and external factors of the organisation. The organisational characteristics which are built on the system, resources and culture form the internal factors which are more influential than the external factors 
on tools and techniques adoption in risk management [15].

Rostami et al. [15] investigated that the tools and techniques adoption is one of the key difficulties of SMEs in the practise of risk management in the construction industry. They outlined that SMEs' knowledge and experience in the process have precedence over organisational constraints. This emphasised the viewpoints of Chileshe and Kikwasi [16] that the absence of awareness and experience in tools and techniques are two key innate challenges of professionals in managing risk in construction industry. Therefore, assessing the most beneficial tools and techniques helps SMEs in the practise of risk management and improves business management.

A thorough literature review on tools and techniques in risk management specified that the key tools and techniques in risk identification and analysis are: brainstorming, interviews, Delphi, check-lists, hazard analysis and critical control points, environmental risk assessment, structure "what if", scenario analysis, business impact analysis, root cause analysis, failure effect mode analysis, event tree analysis, cause and effect analysis and consequence and probability matrix [7], [17], [18], and [19]. A number of these techniques such as the Structure "What If" (SWIFT), Environmental Risk Assessment and Reliability Centred Maintenance techniques can be implemented and practised in both stages. This study adopts the British Standard [13] type of tools and techniques for risk identification.

The question of major relevance to SMEs considering the practise of risk management is: which tools or techniques can provide the greatest benefits?

\section{Risk Identification Tools and Techniques}

The Risk Identification process, as the initial step of risk management, forms the structure of the whole process [18], [20]. Failure in the identification of risks can cause inadequacy in the whole process, which can in turn critically affect the organisation's resources. This process assists organisations in risk management to: (1) recognise the best and most relevant input data (2) understand the benefits of the process (3) recognise risks and their potential impacts (4) provide information for decision-makers [21], [22], and [23]. The risk identification process or risk information gathering process can be achieved with the aid of different tools and techniques. The most common tools and techniques are: documentation reviews, expert judgment, diagramming techniques, assumption analysis, information gathering, checklists and SWOT technique. [13], [18]

Chapman [18] through the behavioural scientists' framework which was developed by Handy [24], conducted an empirical research on the effectiveness of risk identification and assessment techniques in construction projects. The research divided risk identification tools and techniques into three main categories by the degrees of involvement of people, comprising: identification by expert; one-to-one interview; and working group led by analysts. The result cited that the brainstorming technique which involves the analyst leading a working group is the most widely used risk identification technique in large organisations.

Further, Lyons and Skitmore [7] sought the frequency of use of risk management techniques in the construction engineering industry. Project managers from 44 enterprises highlighted that the checklists, brainstorming, case-based approach and scenario building were the most frequently used tools and techniques for identifying risks. They indicated that the success rate of their projects was highly influenced by the association of techniques.

In organisations, a series of factors are involved in the practice of techniques in risk identification [25]. These factors affect the effectiveness of techniques in achieving their objectives, and include: given, intervening and output factors [18]. The givens embrace the organisation's existing factors which impact outputs. The main given factors comprise resources, leader knowledge and skills, and tools and technologies. The intervening factors are temporary factors which are adopted to improve the productivity or user satisfaction such as leadership and procedure. Finally, the output factors cover the satisfaction and user-expected results in terms of their objectives.

In the context of SMEs, restrictions imposed by organisational given factors, such as finance and technology; force SMEs to adopt cost-effective and time-effective techniques [26]. Hence, working group techniques like brainstorming for risk identification which was highlighted by chapman [18] and Lyons and Skitmore [7], are not appropriate for most SMEs. This paper attempts to identify the most common tools and techniques in the risk identification process, and assesses their efficacy in SMEs in the UK construction industry.

\section{Research Methodology}

This paper aims to identify key tools and techniques in the risk identification process in SMEs in the UK construction industry that can be applied to the development of a framework for risk management process for SMEs. The research methodology in this study includes a comprehensive literature review, data collection and sampling, and data analysis. The data for the study was obtained by means of a postal questionnaire. Organisations which participated in this study employed more than 10 people but less than 250 employees based on European Commission's definition. This research focused on small and medium-sized enterprises without consideration of micro businesses. Database for the study was obtained from the Office of National Statistics (ONS), the Small Business Gateway, the Financial Analysis Made Easy (FAME) and the Scottish Centre for the Built Environment (SCBE). 453 small and medium-sized enterprises participated in this study. Attempts were placed to have samples across 
architecture, engineering and construction organisations which had an adequate level of experience and knowledge in risk management.

Based on existing literature and British Standard [13] a list of appropriate tools and techniques for risk identification were identified. This list included 42 tools and techniques. This provided the opportunity to clarify the content of the literature review results and recognise details behind the key issues. This also added breadth to the research and structured the basis of the questionnaires. The research's questionnaire included two sections. Section one covered the organisations' general information such as size and role in the current or most recent project. Section two discussed tools and techniques from the provided list that were practised in risk identification, and further challenges in their practices.

SMEs were asked to identify and rate the most practiced tools and techniques in risk identification process on five-point scale from "not used at all" to "used to a very large extent". Within the survey participants were also questioned to state the reasons behind the use of the practised tools and techniques. In this part, both organisational and environmental variables were considered. The variables included: uncomplicated processes to set up and practise; easy to understand, for practitioners; time and cost effectiveness; valuable results and outcomes; being familiar, from other management processes; unwillingness to learn and practise new methods; lack of investment in training; lack of investment to replace new methods (Software-Technology); etc.

The research questionnaire was collected by post and electronic mailing system. The data were then transferred to the Excel spread sheets and analysed by adopting Statistical Package for the Social Sciences (SPSS). The determined variables (1) tools and techniques with their rate of practice and (2) reasons for the most commonly practised tools and techniques were considered to conduct factor analysis. Factor analysis is a multivariate statistical approach and is designed for data exploration [27]. This technique helped to condense the set of tools and techniques in risk identification in SMEs through the following steps.

a) Assessment of the suitability of the data for factor analysis

b) Factor extraction

c) Factor rotation and interpretation a) Assessment of the suitability of the data for factor analysis: Prior to factor analysis all variables in the tools and techniques adoption were tested for outliers and factorability. Firstly, the strength of the inter-correlations among the difficulties was assessed by the correlation matrix. According to the SPSS guideline the matrix of correlations should illustrates at least some correlations above 0.3 . Outcomes of the research identified considerable amount of coefficients above 0.3. Secondly, the result of Kaiser-Meyer-Olkin (KMO) test for tools and techniques was 0.847 (Table 1) which is acceptable for factor analysis [28]. Finally, the Bartlett's test of the sphericity outcome in this research is $\rho=.00$ which should be statistically significant at $\rho<.05$ [29] (Table 1). From the test results it was manifested that they all satisfied all the assumptions of the factor analysis.

Table 1. Kaiser-Meyer-Olkin (KMO) and Bartlett's test

\begin{tabular}{|c|c|c|}
\hline \multicolumn{2}{|c|}{$\begin{array}{c}\text { Kaiser-Meyer-Olkin Measure of Sampling } \\
\text { Adequacy }\end{array}$} & .847 \\
\hline \multirow{3}{*}{$\begin{array}{c}\text { Bartlett's Test of } \\
\text { Sphericity }\end{array}$} & Approx. Chi-Square & 726.354 \\
\cline { 2 - 3 } & df & 21 \\
\cline { 2 - 3 } & Sig. & .000 \\
\hline
\end{tabular}

b) Factor extraction: Factor analysis is used to determine the smallest number of factors that are required to represent the interrelationships among the set of variables [30]. This process was obtained by the Principle Components Analysis (PCA) technique. The PCA is the default method in most statistical analysing programmes and is common in factor analysis to identify underlying factors [30]. In this study, two techniques which include: Kaiser's criterion [31], and Scree-plot test [32] were conducted to determine the smallest number of factors that are required for factor analysis.

1) Kaiser's criterion: In Total Variance Explained table from factor analysis (Table 2) in column Initial Eigenvalues the values above 1 highlight the number of factors (4.424 and 1.034). These two factors define the number of factors that are required for factor analysis.

2) Scree-plot test: the straight red line drawn based on smaller eigenvalues in Figure 1 indicates the linearity breakpoint of the graph at component two. The components above the breakpoint are the number of factors which need to be considered in the research.

Table 2. Total variance explained

\begin{tabular}{|c|c|c|c|c|c|c|c|}
\hline \multirow{2}{*}{ Component } & \multicolumn{3}{|c|}{ Initial Eigenvalues } & \multicolumn{3}{|c|}{ Extraction Sums of Squared Loadings } & \multirow{2}{*}{$\begin{array}{c}\text { Rotation Sums of } \\
\text { Squared Loadings } \\
\text { Total }\end{array}$} \\
\hline & Total & $\%$ of Variance & Cumulative \% & Total & $\%$ of Variance & Cumulative \% & \\
\hline 1 & 4.424 & 63.205 & 63.205 & 4.424 & 63.205 & 63.205 & 4.141 \\
\hline 2 & 1.034 & 14.772 & 77.977 & 1.034 & 14.772 & 77.977 & 2.817 \\
\hline 3 & 0.463 & 6.612 & 84.589 & & & & \\
\hline 4 & 0.396 & 5.653 & 90.242 & & & & \\
\hline 5 & 0.353 & 5.041 & 95.283 & & & & \\
\hline 6 & 0.205 & 2.924 & 98.208 & & & & \\
\hline 7 & 0.125 & 1.792 & 100 & & & & \\
\hline
\end{tabular}




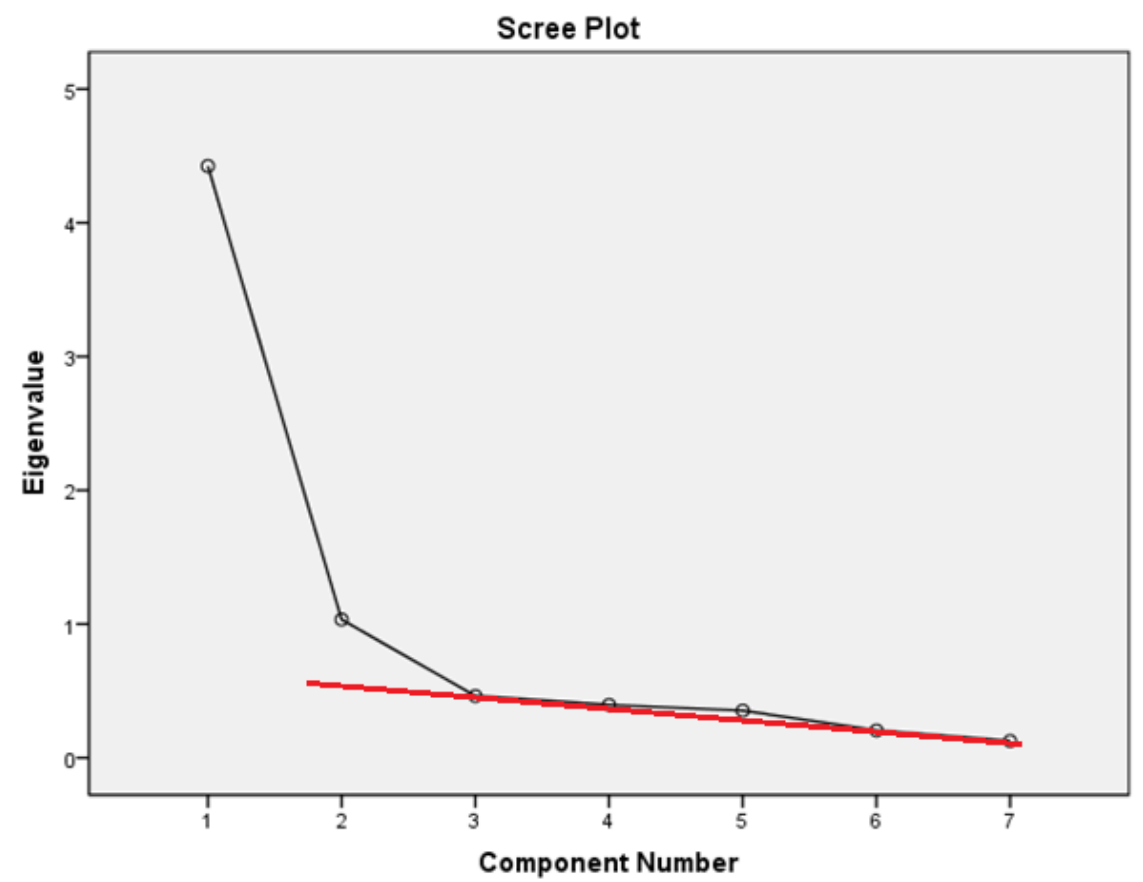

Figure 1. Scree-Plot

Table 3. Pattern and structure matrices for PCA with oblimin rotation of two factor solution of tools and techniques

\begin{tabular}{|c|c|c|c|c|}
\hline \multirow{2}{*}{ Tools and Techniques } & \multicolumn{2}{|c|}{ Pattern coefficients Component } & \multicolumn{2}{|c|}{ Structure coefficients Component } \\
\cline { 2 - 5 } & 1 & 2 & 1 & 2 \\
\hline Documentation review & 0.98 & & 0.932 & 0.454 \\
\hline Expert Judgment & 0.937 & & 0.914 & 0.352 \\
\hline Check list analysis & 0.908 & & 0.891 & 0.416 \\
\hline Information gathering & 0.719 & & 0.895 & 0.596 \\
\hline SWOT & 0.563 & 0.356 & 0.739 & 0.635 \\
\hline Assumption analysis & & 0.86 & 0.472 & 0.883 \\
\hline Diagramming techniques & & 0.838 & 0.414 & 0.881 \\
\hline
\end{tabular}

According to the above techniques two-factor solution for the factor analysis method was considered to extract the most practised tools and techniques in risk identification.

c) Factor rotation and interpretation: The two-factor solution proved a total of $77.98 \%$ of the variance (component 1: 63.21\% and component 2: 14.77\% - Table 2, Cumulative column). To aid in the interpretation of these two components, Oblimin Rotation [33] was performed. This process presented the most practised tools and techniques (highlighted items in Table 3 ).

\section{Discussion of Factor Analysis Results}

From the results, it is evident that the most practised tools and techniques in risk identification are documentation review, expert judgment, check list analysis and information gathering. These results are not in line with the Chapman's [18] studies which specified that brainstorming from the information gathering techniques is the most widely practised technique for risk identification in large construction companies. The results indicate that most SMEs due to their limited resources, knowledge and skills have less capability to implement group-based techniques such as brainstorming.

\subsection{Documentation Review}

The first tool that emerged was documentation review: 441 out of 453 organisations were familiar with this tool and about $75 \%$ of them considered the tool as the first preferred tool to identify risks of the organisation. Collecting data by reviewing the existing documents is known as the documentation review technique. This review contains documents of the previous business plans, strategies, activities, contracts and other stored information in either hard copy or electronic formats. The technique is used to collect background information to understand and identify the new business probabilities and uncertainties [34]. This review assists to recognise the strengths and 
weaknesses of the organisation and understand the history and philosophy of the business. SMEs highlighted that the technique helped them to develop the risk identification process by assessing the organisation's existing documents to design focus groups or an observation framework.

The participants explained that this technique included the assessment of the existing documents at the start of the process which was to find out what types of information were available and which part(s) of them were required for further review. The assessment step is followed by the compiling process which specifies the limit of the review to answer the evaluation questions. Further, accuracy of the compiled information needs to be checked by the management team. In this step the usable part of the information is criticised to derive the key information out of the documents.

\subsection{Expert Judgment}

The second risk identification technique that emerged from 397 questionnaires with $71 \%$ usage rate among SMEs was expert judgment. The expert judgment tool is widely practiced in different stages of the businesses and potentially covers both internal and external risks. The SMEs stated that the expert judgment was implemented and practiced because of: the affordability of resources required in terms of time and budget; valuable results and outcomes; uncomplicated process to set up and quick to produce results. They noted that the outcomes of this technique could be as accurate as other costly tools and techniques like the diagramming techniques.

The expert judgement is a process which is founded on the knowledge and experiences of individuals or groups. People with specialised knowledge, either part of the organisation or involved in a specific activity of the business, are known as the experts of the business [35]. The research revealed that the expert judgment in SMEs highly relies on the experiences and skills of the SMEs' owners and managers. 87 companies indicated that they used their managerial experiences due to their familiarity with the business activities instead of the costly advice of consultants. Two small organisations argued that if the firm could hire a part-time consultant with relevant expertise, knowledge and skills then perhaps they could operate the business with more success probability (fewer risks). They believed that utilising the skills of an expert would be more beneficial and practical than business management analysis. A business manager from a medium-sized company highlighted that the efficacy of the expert judgement technique highly depends on both internal and external experiences. The risks identified by the management team should be reviewed by a consultant with relevant experience of similar business areas to obtain the best possible outcomes.

\subsection{Checklist Analysis}

The third technique that emerged was checklist analysis which was familiar to 385 organisations. $68 \%$ of them stated that this technique was currently being practiced in their business. This technique is known as a basic method of risk identification in which pre-identified threats and opportunities are investigated for signs of potential risk situations [36], [37] and [38]. Checklists within organisation are developed over time through functional experts' contributions and collective experiences [39].

Five medium-sized and three small-sized enterprises named the checklist as the starter of the risk identification which gradually forms the structure of the process. Checklist helps to speed up the whole process and stops organisations from forgetting the critical steps due to disruptions. A medium-sized company presented the checklist as the source of the Risk Breakdown Structure (RBS) which supports the team in better understanding of involved risks. This company analogised the RBS with the Work Breakdown Structure (WBS) in mapping the details of their activities. More than $25 \%$ of participants noted the lack of investment in training and technology as the main barriers to replacing alternative (new) methods with old-fashioned techniques such as checklist analysis.

\subsection{Information Gathering}

The forth common set of techniques which were practiced in more than $61 \%$ of the SMEs was information gathering. 372 out of 453 organisations were familiar with the information gathering techniques. They indicated that the process of information gathering helps to enhance the organisation's memory, develop effective management and save resources. The most important techniques in this method include interviewing, brainstorming, Delphi technique and root cause analysis. 36 participants named the interviewing and brainstorming techniques as the most utilised information gathering techniques in risk identification.

The risk identification process through the interviewing technique can be an individual assessment or involve a group of people [40].This technique is categorised as a resource-intensive technique due to its requirement of organisational resources and time. The collected information through this technique is used to provide a ground for further risk identification. Chapman and Ward [4] believed that individual straightforward approaches such as the pondering approach which is based on individual identification assessment could be more beneficial for organisations instead of costly interviews.

The brainstorming is the second popular technique of the information gathering process among construction SMEs. The aim of this technique is to provide a comprehensive list of risks by the business team and multidisciplinary specialists. Osborn [41] introduced the brainstorming as a problem solving method that provides a considerable range of ideas in less time. This technique contains identifying problems, creating ideas, introducing and developing solutions sub-processes [18]. The results of the study 
revealed that the medium-sized participating firms were more likely to implement this technique for being familiar to it as well as its time effectiveness character. Two of those companies indicated that they used semi-structured interviews for the ground phase of the risk identification process and used its generated information for identifying problems in the brainstorming technique.

\section{Difficulties in Practise of Tools and Techniques in Risk Identification}

Chapman [18] outlined that the most effective risk identification tools and techniques were those of the group work. In line with that study, Raz and Michael [25] also confirmed that the brainstorming and periodic risk reporting techniques were the most common risk identification techniques in the software engineering sector, which are regularly adopted by large enterprises. However, the results of the current study revealed the frequent use of individuals' tools and techniques such as documentation review, expert judgment and checklist analysis among the SMEs in the UK construction industry.

The results highlight that the adoption of a tool or technique is influenced by the size of the organisation. In SMEs for high degree of centralisation in management and low level of complexity, individual factors such as personality, aptitude, experience, knowledge and leadership have more impact than organisational factors [42]. The individual factors generate an organic structure with informal working relationships which restrict activities and collaborations within organisations [43]. Also, limitations imposed by organisational factors; i.e. resources and technologies, force SMEs towards economical practices [26] and [44]. Therefore, group work techniques such as brainstorming are not suitable for most small and medium-sized organisations.

Through the research questionnaire, SMEs indicated that factors such as uncomplicated process to set up and practise; easy to understand for practitioners; time and cost effectiveness; valuable results and outcomes; being familiar to other management processes; lack of investment in training to learn alternative methods, and low degree of budget to replace new methods are the main reasons for the practise of the highlighted (individual) techniques.

Participants outlined the documentation review as the most appropriate technique for risk identification. This technique was identified as the most cost and time effective technique because of its independent character and uncomplicated process. However, the quality of information being gathered through this technique should be assessed together with the outcomes of other information collecting techniques such as interviews, questionnaires and checklists in the risk identification process.

\section{Limitation}

The research provides a range of notable contributions to risk identification in both theory and practice; however, several limitations of the study need to be acknowledged. This study considered organisations as small or medium-sized enterprises and did not distinguish them based on their size. The size of organisations forms organisational characteristics which can be affect initiation and implementation of tools and techniques in risk identification. Small-sized enterprises with less amount of resources and technology have more barriers in use of tools and techniques in the risk identification process. Moreover, in terms of field of practice within the construction industry the study did not classify enterprises i.e. contractors, engineers, and architectures. Their field of practice can influence their selection of tools and techniques. Second, the study addressed the UK construction enterprises; hence, the results may not directly apply to other industries or similar organisation operating in other countries. The third limitation is associated with the method of the study which is based on quantitative analysis. A qualitative approach with more detailed questions could provide different results by addressing multiple class sections.

\section{Conclusions}

Risk Identification, as the initial step of risk management, forms the structure of the whole process. Failure in the identification of risks can cause inadequacy in the whole process which can in turn critically affect the organisation's resources. Firms that have implemented risk management have recognised that there would be a higher probability of failure if appropriate tools and techniques are not carefully employed. The adopted tools and techniques in risk identification determine the accuracy of the results of the risk management process. Therefore, on the basis of a quantitative survey with the UK construction enterprises, four key tools and techniques were identified on an assessment of their likelihood of usage and degree of efficacy in risk identification. The documentation review, expert judgment, checklist analysis and information gathering were highlighted to have significant impacts on the efficiency of risk identification. The attempt to evaluate these key tools and techniques from the perspective of organisational characteristics indicated that valuable results and outcomes; time and cost effectiveness; and uncomplicated processes are the main reasons of their practice within small and medium sized enterprises. Conversely, the group-based techniques like brainstorming because of SMEs' inadequate level of knowledge and resources are less practised. Findings of the study can be applied to the development of a framework for the risk management process for small and medium-sized enterprises. 


\section{REFERENCES}

[1] Global Construction Perspectives and Oxford Economics, Online available from http://www.globalconstruction 2025.com/uk/

[2] E. A. A. Mlybari, Managing value, requirements and risk in the appraisal stage of UK construction projects, $\mathrm{PhD}$ Thesis, University of Leeds, School of Civil Engineering, 2011.

[3] S. Z. S. Tabish and K. N. Jha, Identification and evaluation of success factors for public construction projects, Construction Management and Economics, Vol. 29, No. 8, pp. 809-823, 2011.

[4] C. Chapman and S. Ward, Project Risk Management: Processes, Techniques and Insights, 2nd ed, John Wiley, p.131, 2008.

[5] British Standard (BS 31000), BSI British Standard, Risk Management-Code of Practice, 2009.

[6] N. J. Smith, T. Merna and P. Jobling, Managing risk in construction projects, John Wiley and Sons, 3rd Edition, 2014.

[7] T. Lyons and M. Skitmore, Project Risk Management in the Queensland Engineering Construction Industry: A survey, International Journal of Project Management, Vol. 22, No. 1, pp. 51-61, 2004.

[8] S. Kim and D. Bajaj, Risk management in construction: an approach for contractors in South Korea, Cost Engineering, Vol. 42, No. 1, pp. $38-44,2000$.

[9] Y. Frimpong, J. Oluwoye and L. Crawford, Causes of delay and cost overruns in construction of groundwater projects in a developing countries; Ghana as a case study, International Journal of Project Management, Vol. 21, Issue 5, pp. 321-326, 2003.

[10] C. B. Chapman, Risk analysis and inherent risk management priorities. In: Collaboration Management: New Project and Partnering Techniques, pp. 107-128. John Wiley and Sons, Chichester, 1994.

[11] J. Couillard, The role of project risk in determining project management approach, Project Management Journal, Vol. 26, No. 4, pp. 3-15, 1995.

[12] Y. A. Debrah and G. Ofori, Emerging managerial competencies of professionals in the Tanzanian construction industry, The International Journal of Human Resource Management, Vol. 16, No. 8, pp. 1399-1414, 2005.

[13] British Standard (BS 31010), BSI British Standard, Risk Management, Risk assessment techniques, 2010.

[14] T. O. Lee and C. A. Wilhelmsen, Risk Assessment: Tools, Techniques, and Their Applications, John Wiley and Sons, p. $416,2012$.

[15] A. Rostami, J. Sommerville, I. Wong and C. Lee, Risk Management Implementation in Small and Medium Enterprises in the UK Construction Industry, Engineering, Construction and Architectural Management, Vol. 22, No. 1, pp. 91-107, 2015.
[16] N. Chileshe and G. J. Kikwasi, Perception of barriers to implementing risk assessment and management practices by construction professionals in Tanzania, 29th Annual ARCOM Conference, pp. 1137-1146, Reading, UK, 2013.

[17] A. S. Akintoye and M. J. MacLeod, Risk analysis and management in construction, International Journal of Project Management, Vol. 15, No. 1, pp. 31-38, 1997.

[18] R. J. Chapman, The effectiveness of working group risk identification and assessment techniques, International Journal of Project Management, Vol. 16, Issue 6, pp. 333-343, 1998.

[19] S. Baker, D. Ponniah and S. Smith, Risk response techniques employed currently for major projects, Construction Management and Economics, London, Vol. 7, No. 2, pp. 205-213, 1999.

[20] S. C. Ward and C. Chapman, Transforming project risk management into project uncertainty management, International Journal of Project Management, Vol. 21 (2003), pp. 97-105, 2003.

[21] K. Simu, Risk Management in Small Construction Projects, Licentiate Thesis, Lulea University of Technology, Department of Civil and Environmental Engineering, 2006.

[22] T. Henschel, Risk Management Practices in the Main Industries of German Small to Medium-Sized Enterprises, An Empirical Investigation, 2007.

[23] C. Wang, E. A. Walker and J. L. Redmond, Explaining the lack of strategic planning in SMEs: The importance of owner motivation, International Journal of Organisational Behaviour, Vol. 12, No. 1, pp. 1-16, 2007.

[24] C. B. Handy, Understanding organisations, Penguin Books Limited, 4th edition, Middlesex, UK, 1993.

[25] T. Raz and E. Michael, Use and Benefits of Tools for Project Risk Management, International Journal of Project Management, Vol. 19 (2001), pp. 9-17, 2001.

[26] J. Welsh and J. White, A small business is not a little big business, Harvard Business Review, pp. 18-32, 1981.

[27] B. Williams, A. Onsman and T. Brown, Exploratory factor analysis: A five-step guide for novices, Journal of Emergency Primary Health Care (JEPHC), Vol. 8, Issue 3, Article 990399 , 2010.

[28] B. G. Tabachnick and L. S. Fidell, Using Multivariate Statistics, Boston: Pearson Education, 5th Edition, 2007.

[29] M. S. Bartlett, A note on the multiplying factors for various chi square approximations, Journal of the Royal Statistical Society, Vol. 16 (Series B), pp. 296-8, 1954.

[30] B. Thompson, Exploratory and confirmatory factor analysis: understanding concepts and applications, American Psychological Association, Washington, DC, 2004.

[31] H. Kaiser, The application of electronic computers to factor analysis, Educational and Psychological Measurement, Vol. 20, No. 1, pp. 141-51, 1960.

[32] R. B. Cattell, The Scree Test for Number of Factors, Multivariate Behavioural Research, Vol. 1, Issue 2, pp. 245-276, 1966.

[33] A. B. Costello and J. W. Osborne, Best Practices in 
Exploratory Factor Analysis: Four Recommendations for Getting the Most from Your Analysis, Practical Assessment, Research \& Evaluation, Vol. 10, No. 7, pp. 1-9, 2005.

[34] B. R. Witkin and J. W. Altschuld, Planning and Conducting Needs Assessments: A Practical Guide, Sage Publications, Inc, 1995.

[35] H. Otway and D. V. Winterfeldt, Expert Judgment in Risk Analysis and Management: Process, Context, and Pitfalls. Risk Analysis, Vol. 12, No. 1, pp. 83-93, 1992.

[36] W. R. Duncan, A Guide to the Project Management Body of Knowledge, Project Management Institute, Newtown Square, PA, pp. 111-21, 1996.

[37] H. Kumamoto and E. J. Henley, Probabilistic Risk Assessment and Management for Engineers and Scientists, IEEE Press, Piscataway, NJ, 1996.

[38] J. Cross, Lecture Notes for SESC9211: Risk Management, School of Safety Science, The University of New South Wales, Sydney, 2001.
[39] S. C. Ward, Assessing and managing important risks, International Journal of Project Management, Vol. 17, No. 6, pp. 331-6, 1999.

[40] R. J. Chapman, The Controlling Influences on Effective Risk Identification and Assessment for Construction Design Management, International Journal of Project Management, Volume 19, Issue 3, pp. 147-160, 2001.

[41] A. F. Osborn, Applied Imagination, Principles and Procedures of Creative Problem Solving, third revised edition, fourteenth printing Charles Scribner's Sons, New York, 1963.

[42] A. Ghobadian and D. Gallear, "TQM and Organisation Size", International Journal of Operations and Production Management, Vol. 17, No. 2, pp. 121-163, 1997.

[43] T. Burns and G. M. Stalker, The Management of Innovation, 2nd Edition, Tavistock Publishing, London, 1966.

[44] G. D'Amboise and M. Muldowney, "Management Theory for Small Business: Attempts and Requirements." The Academy of Management Review, Vol. 13, No. 2, pp. 226-240, 1988. 